\title{
Scalar Conservation Laws with Vanishing and Highly Nonlinear Diffusive-Dispersive Terms
}

\author{
By
}

Naoki FuJINO*

\begin{abstract}
We investigate the initial value problem for a scalar conservation law with highly nonlinear diffusive-dispersive terms: $u_{t}+f(u)_{x}=\varepsilon\left(u_{x}^{2 \ell-1}\right)_{x}-\delta\left(u_{x}^{2 \ell-1}\right)_{x x}(\ell \geq 1)$. In this paper, for a sequence of solutions to the equation with initial data, we give convergence results that a sequence converges to the unique entropy solution to the hyperbolic conservation law. In particular, our main theorem implies the results of Kondo-LeFloch [15] and Schonbek [26], furthermore makes up for insufficiency of the results in Fujino-Yamazaki [9] and LeFloch-Natalini [22]. Applying the technique of compensated compactness, the Young measure and the entropy measure-valued solutions as main tools, we establish the convergence property of the sequence. The final step of our proof is to show that the measure-valued mapping associated to the sequence of solutions is reduced to an entropy solution and this step is mainly based on the approach of LeFloch-Natalini [22].
\end{abstract}

\section{$\S 1$. Introduction and the Main Result}

Consider the sequence $\left\{u^{\varepsilon}\right\}$ of smooth solutions of the Cauchy problem for a scalar conservation law in one space dimension with highly nonlinear diffusive-dispersive terms:

(1.1) $\partial_{t} u+\partial_{x} f(u)=\varepsilon \partial_{x}\left(\partial_{x} u\right)^{2 \ell-1}-\delta \partial_{x}^{2}\left(\partial_{x} u\right)^{2 \ell-1}, \quad(x, t) \in \mathbf{R} \times(0, \infty)$,

$$
u(x, 0)=u_{0}^{\varepsilon}(x), \quad x \in \mathbf{R},
$$

Communicated by H. Okamoto. Received December 20, 2006. Revised March 9, 2007. 2000 Mathematics Subject Classification(s): 35L65, 35L70, 35L75.

Key words: Scalar conservation laws, Entropy solutions, Young measure, Measure-valued solutions.

* Graduate School of Mathematical Sciences, University of Tokyo, Tokyo 153-8914, Japan and Graduate School of Pure and Applied Sciences, University of Tsukuba, Ibaraki 3058571, Japan.

(C) 2007 Research Institute for Mathematical Sciences, Kyoto University. All rights reserved. 
where $\ell \geq 1$ and $\varepsilon, \delta=\delta(\varepsilon) \rightarrow 0+$. In this paper, we will show that the sequence $\left\{u^{\varepsilon}\right\}$ converge to the unique entropy solution to

$$
\begin{aligned}
u_{t}+f(u)_{x} & =0, \quad(x, t) \in \mathbf{R} \times(0, \infty), \\
u(x, 0) & =u_{0}(x), \quad x \in \mathbf{R} .
\end{aligned}
$$

To obtain the convergence property of $\left\{u^{\varepsilon}\right\}$, we assume that there exist the smooth solutions to Eqs. (1.1) and (1.2) defined on $\mathbf{R} \times\left(0, T^{*}\right)$ for some $T^{*}>0$, vanishing at infinity and associated with smooth and compactly supported initial data $u_{0}^{\varepsilon}$ and

$$
\exists u_{0} \in L^{1}(\mathbf{R}) \cap L^{q}(\mathbf{R}), \forall q>1 \text { s.t. } \lim _{\varepsilon \rightarrow 0} u_{0}^{\varepsilon}=u_{0} \text { in } L^{1}(\mathbf{R}) \cap L^{q}(\mathbf{R}) .
$$

In addition, we assume the following uniform boundedness concerning the initial data with some constant $C_{0}>0$ independent of $\varepsilon$ :

$$
\left\|u_{0}^{\varepsilon}\right\|_{L^{2}(\mathbf{R})}+\left\|u_{0}^{\varepsilon}\right\|_{L^{q}(\mathbf{R})}+\delta^{\frac{1}{2 \ell}}\left\|u_{0, x}^{\varepsilon}\right\|_{L^{2 \ell}(\mathbf{R})} \leq C_{0},
$$

for $q \in\left(\frac{3 \ell-1}{\ell}, \frac{3 \ell^{2}+2 \ell-1}{\ell}\right)(\ell \geq 1)$.

On the other hand, we also assume for the flux function $f(u)$ that $f(u)$ is a given smooth function which satisfies the following growth condition:

$$
\exists C_{1}>0, m>1 \text { s.t. }\left|f^{\prime}(u)\right| \leq C_{1}\left(1+|u|^{m-1}\right) \text { for any } u \in \mathbf{R} .
$$

When $F$ is defined by $F^{\prime}(u)=f(u)$, we can replace the condition (I) by an assumption (I'):

$$
\exists C_{2}>0, m>1 \text { s.t. }|F(u)| \leq C_{2}\left(|u|^{2}+|u|^{m+1}\right) \text { for any } u \in \mathbf{R} .
$$

Then, under the above assumptions, we show the following main result of the present paper:

Theorem 1.1. Suppose that a condition (I) and there exists a sequence $\left\{u^{\varepsilon}\right\}$ of the smooth solutions to Eqs. (1.1) and (1.2) defined on $\mathbf{R} \times\left(0, T^{*}\right)$, vanishing at infinity and associated with the initial data satisfying (1.5) and (1.6) with $m<q\left(q \in\left[\frac{3 \ell^{2}+3 \ell-1}{2 \ell}, 3 \ell\right]\right)$. If $\delta=O\left(\varepsilon^{\frac{(\ell+1)(6 \ell-m-1)}{2\left(3 \ell^{2}-m \ell-q \ell+3 \ell-1\right)}}\right)$, then the sequence $\left\{u^{\varepsilon}\right\}$ converge to the unique entropy solution $u \in L^{\infty}\left(0, T^{*} ; L^{q}(\mathbf{R})\right)$ to Eqs. (1.3) and (1.4) in $L^{k}\left(0, T^{*} ; L^{p}(\mathbf{R})\right)(\forall k<\infty$ and $\forall p<q)$.

Moreover if we assume that the sequence $\left\{u^{\varepsilon}\right\}$ of solutions to Eqs. (1.1), (1.2) is bounded in $L^{\infty}\left(0, T^{*} ; L^{q}(\mathbf{R})\right)$, we obtain the same conclusion for any $q>m(m \in(1,3 \ell), \ell \geq 1)$ provided that $\delta=o\left(\varepsilon^{\frac{(\ell+1)(6 \ell-m-1)}{2 \ell(3 \ell-m)}}\right)$. Namely it follows that 
Theorem 1.2. $\quad$ Suppose that a condition (I) and there exists a sequence $\left\{u^{\varepsilon}\right\}$ of the smooth solutions to Eqs. (1.1) and (1.2) defined on $\mathbf{R} \times\left(0, T^{*}\right)$, vanishing at infinity and associated with the initial data satisfying (1.5) and (1.6). If the sequence is uniformly bounded in $L^{\infty}\left(0, T^{*} ; L^{q}(\mathbf{R})\right)$ for some $q>m$ $(m \in(1,3 \ell))$ and $\delta=o\left(\varepsilon^{\frac{(\ell+1)(6 \ell-m-1)}{2 \ell(3 \ell-m)}}\right)$, then the sequence $\left\{u^{\varepsilon}\right\}$ converge to the unique entropy solution $u \in L^{\infty}\left(0, T^{*} ; L^{q}(\mathbf{R})\right)$ to Eqs. (1.3) and (1.4) in $L^{k}\left(0, T^{*} ; L^{p}(\mathbf{R})\right)(\forall k<\infty$ and $\forall p<q)$.

In the consideration of a convergence, the appropriate balance in the relation between $\varepsilon$ and $\delta$ is claimed so that the sequence of solutions to the conservation laws with diffusion and dispersion terms converges to the solution to the hyperbolic conservation law. In fact, when $\delta=0$, Eq. (1.1) is reduced to a parabolic equation. In this case, if $\varepsilon \rightarrow 0$ (with $\delta=0$ ), it is rather trivial that the sequence $\left\{u^{\varepsilon}\right\}$ of solutions to Eq. (1.1) converges to the solution to (1.3) owing to the classical vanishing viscosity method. On the other hand, when $\varepsilon=0$, then Eq. (1.1) is reduced to the generalized Korteweg-de Vries $(\mathrm{KdV})$ equation [16]. If $\delta \rightarrow 0$ in the $\mathrm{KdV}$ equation, the sequence of the solutions to Eq. (1.1) does not converge to the solution to Eq. (1.3) in general (cf. $[3,4,19,20])$.

We recall several fundamental results for the convergence problem to the scalar conservation laws with diffusion and dispersion terms:

$$
\partial_{t} u^{\varepsilon}+\partial_{x} f\left(u^{\varepsilon}\right)=R^{\varepsilon}, \quad u^{\varepsilon}=u^{\varepsilon}(x, t)
$$

where $\varepsilon>0$ and $R^{\varepsilon}=R^{\varepsilon}\left(u^{\varepsilon}, u_{x}^{\varepsilon}, u_{x x}^{\varepsilon}, \cdots\right) \rightarrow 0$ as $\varepsilon \rightarrow 0$. In particular, our main Theorem 1.1 includes previous results. For the linear diffusion and the linear dispersion terms as $\ell=1$ in Eq. (1.1) i.e.

$$
R^{\varepsilon}=\varepsilon u_{x x}^{\varepsilon}-\delta u_{x x x}^{\varepsilon}
$$

a first convergence result is obtained by Schonbek [26] under the assumption that either $\delta=O\left(\varepsilon^{2}\right)$ for Burgers' type flux $\left(f(u)=\frac{u^{2}}{2}\right)$ and for the family of flux functions:

$$
f(u)=-\frac{u^{2 h+1}}{2 h+1}, \quad h \geq 1,
$$

or the stronger condition $\delta=O\left(\varepsilon^{3}\right)$ for general subquadratic flux functions $f$. This convergence result has been improved by Kondo-LeFloch [15] for the flux satisfying $\left|f^{\prime}(u)\right| \leq M$ (for $\forall u \in \mathbf{R}, M>0$ ). They give that the subsequence of solutions converges in $L^{k}\left(0, \infty ; L^{p}(\mathbf{R})\right)(1<k<\infty$ and $1<p<2)$ to a weak solution of the Cauchy problem (1.3), (1.4) under the assumption $\delta=O\left(\varepsilon^{2}\right)$. 
Moreover they obtain that the limit is the unique entropy solution in the sense of Kružkov under the stronger condition $\delta=o\left(\varepsilon^{2}\right)$. They also give a convergence result for multidimensional conservation laws. Clearly, our result is extension to their works. See also a result for systems in Hayes-LeFloch [11].

As compared with the above results, there are the following results for the nonlinear diffusion and the nonlinear dispersion terms:

$$
R^{\varepsilon}=\varepsilon b\left(u_{x}^{\varepsilon}\right)_{x}-\delta\left(\left(u_{x}^{\varepsilon}\right)^{2 \ell-1}\right)_{x x}, \quad \ell \geq 1
$$

under the assumptions that $f$ satisfies the growth condition (I) and moreover that a nondecreasing function $b$ satisfies $b(0)=0, b(\lambda) \lambda \geq 0$ (for $\forall \lambda \in \mathbf{R}$ ) and

$$
C_{3}|\lambda|^{(2 \ell+1) r} \leq b(\lambda) \lambda \leq C_{4}|\lambda|^{(2 \ell+1) r} \text { for any }|\lambda| \geq N
$$

where $C_{3}, C_{4}, N>0, r \geq 1$. In the case as $\ell=1$ in Eq. (1.7), LeFlochNatalini [22] show that the sequence $\left\{u^{\varepsilon}\right\}$ is bounded in $L^{\infty}\left(0, T^{*} ; L^{q}(\mathbf{R})\right)$ for $m<5-\frac{1}{r}(=: q)$ and obtain the convergence result that the sequence converges to the unique entropy solution $u \in L^{\infty}\left(0, T^{*} ; L^{q}(\mathbf{R})\right)$ in $L^{k}\left(0, T^{*} ; L^{p}(\mathbf{R})\right)(k<$ $\infty, p<q)$ for $\delta=O\left(\varepsilon^{\frac{5-m}{r(5-m)-1)}}\right)(r \geq 1)$. In the case that $\ell \geq 1$ for Eq. (1.7), it is investigated by Fujino-Yamazaki [9]. In [9], we prove the same convergence property to [22] for $\delta=O\left(\varepsilon^{\frac{6 \ell-m-1}{r(6 \ell-m-1)-1}}\right)(m<q, \forall \ell \geq 1)$. On the consideration to Eq. (1.7), the assumption (II) of the diffusion term is very important in the proof of their results in $[9,22]$. From the assumption (II), the function $b$ can not imply the identity function $b(\lambda)=\lambda$ because, as $\ell, r=1$ in (II), it follows that

$$
C_{3}^{\prime}|\lambda|^{2} \leq b(\lambda) \leq C_{4}^{\prime}|\lambda|^{2} \text { for any }|\lambda| \geq N
$$

where $C_{3}^{\prime}, C_{4}^{\prime}, N>0$. Comparing with this assumption for $b$ in Eq. (1.7), the nonlinear power function $u_{x}^{2 \ell-1}(\forall u \in \mathbf{R})$ of the diffusion term in our scalar conservation law (1.1) imply the identity function as $\ell=1$ clearly. On the other hand, observing the domain of $q$ for the $L^{q}(\mathbf{R})$, it is that $(m<) q \in[4,5)$ in $[9,22]$ and that $(m<) q \in\left[\frac{3 \ell^{2}+3 \ell-1}{2 \ell}, 3 \ell\right](\ell \geq 1)$ in Theorem 1.1 of this paper. Therefore, in that sense, we can also consider the different results for $q>m$.

In this paper, we consider the scalar conservation laws with highly nonlinear diffusive-dispersive terms (1.1) without the assumptions (II) nor (II') by using the technique developed in [9, 22]. Especially, we make use of the compensated compactness, the measure-valued (m.-v.) solutions of the Cauchy problem which are investigated by, for example, DiPerna [8] and Szepessy [27]. Moreover the final step of the proof of the main result relies mainly on the 
approach of LeFloch-Natalini [22]. To give convergence results Theorems 1.1, 1.2 , we recall some elementary notions in Section 2 and we establish the uniform boundedness in $L^{q}(\mathbf{R})$ by a priori estimates of the solutions to Eq. (1.1) in Section 3. In the last section, owing to a priori estimates and boundedness obtained in Section 3, the convergence argument due to [22] is applied to Eqs. (1.1), (1.2).

\section{§2. Preliminaries}

Let us remind of the basic theory for Young measure and entropy measurevalued (m.-v.) solutions concisely. Following DiPerna [8], LeFloch-Natalini [22] and Szepessy [27], we state a generalization of the Young measure.

Proposition $2.1([8,22,27])$. Suppose that the sequence $\left\{u^{\varepsilon}\right\}$ is bounded in $L^{\infty}\left(0, \infty ; L^{q}(\mathbf{R})\right)$ and that $f \in C(\mathbf{R})$ satisfies the growth condition (I) for some $q^{\prime} \in(0, q), C>0$. Then there exists a subsequence $\left\{u^{\varepsilon^{\prime}}\right\}$ and a probability measure-valued mapping $\nu=\nu_{(x, t)}$ defined on $\mathbf{R} \times(0, \infty)$, such that

$$
f\left(u^{\varepsilon^{\prime}}\right) \rightarrow\left\langle\nu_{(x, t)}, f\right\rangle:=\int_{\mathbf{R}} f(\lambda) d \nu_{(x, t)}(\lambda) \text { as } \varepsilon^{\prime} \rightarrow 0
$$

in $L^{s}(\mathbf{R} \times(0, \infty))$ for any $s \in\left(1, q / q^{\prime}\right)$.

A probability measure-valued mapping $\nu$ in Proposition 2.1 is called a Young measure associated with the subsequence $\left\{u^{\varepsilon^{\prime}}\right\}$. For this Young measure $\nu$, an entropy measure-valued (m.-v.) solution is defined as follows:

Definition 2.1 $([8,27])$. Suppose that $f \in C(\mathbf{R})$ satisfies the growth condition (I) and the initial data $u_{0} \in L^{1}(\mathbf{R}) \cap L^{q}(\mathbf{R})$. If it follows that

$$
\partial_{t}\left\langle\nu_{(x, t)}(\lambda),|\lambda-k|\right\rangle+\partial_{x}\left\langle\nu_{(x, t)}(\lambda), \operatorname{sgn}(\lambda-k)(f(\lambda)-f(k))\right\rangle \leq 0
$$

in $\mathcal{D}^{\prime}(\mathbf{R} \times(0, \infty))$ for any $k \in \mathbf{R}$ and that

$$
\lim _{T \rightarrow 0^{+}} \frac{1}{T} \int_{0}^{T} \int_{K}\left\langle\nu_{(x, t)}(\lambda),\left|\lambda-u_{0}(x)\right|\right\rangle d x d t=0
$$

for any compact sets $K \subseteq \mathbf{R}$, then a Young measure $\nu: \mathbf{R} \times(0, \infty) \rightarrow \operatorname{Prob}(\mathbf{R})$ associated with the subsequence $\left\{u^{\varepsilon^{\prime}}\right\}$ is called an entropy measure-valued (m.v.) solution to Eqs. (1.3), (1.4).

Here we remark that it is not necessary to take a subsequence of $\left\{u^{\varepsilon}\right\}$. As a well-known fact, for an entropy m.-v. solution to Eqs. (1.3), (1.4), uniqueness 
holds by [27]. Namely if $\nu$ and $\tilde{\nu}$ are entropy m.-v. solutions to Eqs. (1.3), (1.4), then there exists a function $w \in L^{\infty}\left(\mathbf{R} ; L^{1}(\mathbf{R}) \cap L^{q}(\mathbf{R})\right)$ such that $\nu_{(x, t)}=$ $\delta_{w(x, t)}=\tilde{\nu}_{(x, t)}$ for a.e. $(x, t) \in \mathbf{R} \times(0, \infty)$. This uniqueness of the entropy m.-v. solution implies $f\left(u^{\varepsilon}\right) \rightarrow\left\langle\nu_{(x, t)}, f\right\rangle$ in the sense of distributions. We introduce the convergence theorem as our main tool.

Theorem 2.1 ([22]). Suppose that $f$ satisfies the growth condition (I) and the initial data $u_{0} \in L^{1}(\mathbf{R}) \cap L^{q}(\mathbf{R})$ for $q \geq 1$. Let $\nu$ be a Young measure associated with $\left\{u^{\varepsilon}\right\}$ which is an uniformly bounded sequence in $L^{\infty}\left(0, \infty ; L^{q}(\mathbf{R})\right)$. If a Young measure $\nu$ is an entropy m.-v. solution to Eqs. (1.3), (1.4), then the sequence $\left\{u^{\varepsilon}\right\}$ converge to the unique entropy solution $u \in L^{\infty}\left(0, \infty ; L^{q}(\mathbf{R})\right)$ in $L^{\infty}\left(0, \infty ; L_{\text {loc }}^{q^{\prime}}(\mathbf{R})\right)\left(\right.$ for any $\left.q^{\prime} \in[1, q)\right)$ to Eqs. (1.3), (1.4).

To obtain our convergence results by applying Theorem 2.1, we should show that the uniform boundedness of a sequence $\left\{u^{\varepsilon}\right\}$ in $L^{q}(\mathbf{R})$ holds for $q>m$ and that a Young measure $\nu$ is an entropy m.-v. solution to Eqs. (1.3), (1.4) in the following sections.

\section{$\S 3 . \quad$ A Priori Estimates}

In this section, to establish the $L^{q}$ boundedness, we give several a priori estimates of solutions to a scalar conservation law with highly nonlinear diffusive-dispersive terms (1.1) with initial data $u_{0}^{\varepsilon}$ which are smooth functions with compact support and satisfy the assumptions (1.5) and (1.6). We suppose that there exists a sequence $\left\{u^{\varepsilon}\right\}$ of the smooth solutions to Eqs. (1.1), (1.2) defined on $\mathbf{R} \times\left(0, T^{*}\right)$, vanishing at infinity and associated with initial data $u_{0}^{\varepsilon}$ for some $T^{*} \in(0, \infty]$.

Throughout the calculation of this section and for simplicity, we omit the upper-index $\varepsilon$ and describe $u^{\varepsilon}$ into $u$ and so on. Referring to [9], as a first estimate, we find

Lemma 3.1. For every $T \in\left(0, T^{*}\right)$, We have

$$
\int_{\mathbf{R}} u^{2}(x, T) d x+2 \varepsilon \int_{0}^{T} \int_{\mathbf{R}} u_{x}^{2 \ell}(x, t) d x d t \leq C_{0},
$$

and

$$
\begin{aligned}
& \delta \int_{\mathbf{R}} u_{x}^{2 \ell}(x, T) d x+2 \ell(2 \ell-1) \varepsilon \delta \int_{0}^{T} \int_{\mathbf{R}} u_{x}^{2(2 \ell-2)} u_{x x}^{2} d x d t \\
& \leq C_{0}+2 \ell \int_{\mathbf{R}} F(u(x, T)) d x+2 \ell \varepsilon \int_{0}^{T} \int_{\mathbf{R}} f^{\prime}(u) u_{x}^{2 \ell} d x d t .
\end{aligned}
$$


Proof. Multiplying Eq. (1.1) by $u$ and integrating it in space, we find

$$
\int_{\mathbf{R}}\left(\frac{u^{2}}{2}\right)_{t} d x=-\varepsilon \int_{\mathbf{R}} u_{x}^{2 \ell} d x
$$

Integrating the above equation in time, We obtain

$$
\frac{1}{2} \int_{\mathbf{R}} u^{2}(x, T) d x+\varepsilon \int_{0}^{T} \int_{\mathbf{R}} u_{x}^{2 \ell} d x d t=\frac{1}{2} \int_{\mathbf{R}} u_{0}^{2}(x) d x .
$$

From an assumption (1.6) of the initial data in $L^{2}$ norm, We arrive the first estimate (3.1).

In the same way, multiplying Eq. (1.1) by $f(u)+\delta\left(u_{x}^{2 \ell-1}\right)_{x}$ and integrating it in space, We have

$$
\int_{\mathbf{R}} F(u)_{t} d x-\delta \int_{\mathbf{R}}\left(\frac{u_{x}^{2 \ell}}{2 \ell}\right)_{t} d x=-\varepsilon \int_{\mathbf{R}} f^{\prime}(u) u_{x}^{2 \ell} d x+(2 \ell-1) \varepsilon \delta \int_{\mathbf{R}}\left(u_{x}^{2 \ell-2} u_{x x}\right)^{2} d x .
$$

By integrating in time, We obtain

$$
\begin{aligned}
& \delta \int_{\mathbf{R}} u_{x}^{2 \ell} d x+2 \ell(2 \ell-1) \varepsilon \delta \int_{0}^{T} \int_{\mathbf{R}}\left(u_{x}^{2 \ell-2} u_{x x}\right)^{2} d x d t \\
& =\delta \int_{\mathbf{R}} u_{0, x}^{2 \ell} d x-2 \ell \int_{\mathbf{R}} F\left(u_{0}\right) d x+2 \ell \int_{\mathbf{R}} F(u) d x+2 \ell \varepsilon \int_{0}^{T} \int_{\mathbf{R}} f^{\prime}(u) u_{x}^{2 \ell} d x d t
\end{aligned}
$$

Hence We obtain an inequality (3.2) by the uniform bound of $u_{0, x}$ in the $L^{2 \ell}$ norm.

Combining an assumption (I') and the uniform bound of $u$ in $L^{\infty}\left(0, T^{*}\right.$; $\left.L^{2}(\mathbf{R})\right)$ derived by a estimate (3.1), we can replace by a following assumption;

(I") $\exists C_{5}>0, m>1$ s.t. $|F(u)| \leq C_{5}\left(1+|u|^{m+1}\right)$ for any $u \in \mathbf{R}$.

To estimate the solution $u$ to Eq. (1.1) in the $L^{\infty}$ norm, we use the estimates (3.1), (3.2) and an assumption (I").

Lemma 3.2. Suppose $m \in(1,6 \ell-1)(\ell \geq 1)$, then there exists a constant $C>0$ such that

$$
\sup _{t \in\left(0, T^{*}\right)}\|u(\cdot, t)\|_{L^{\infty}(\mathbf{R})} \leq C \delta^{-\frac{1}{6 \ell-m-1}} .
$$

Proof. From the inequality (3.2) and an assumption (I"), we have

$$
\begin{aligned}
& \delta \int_{\mathbf{R}} u_{x}^{2 \ell}(x, T) d x+2 \ell(2 \ell-1) \varepsilon \delta \int_{0}^{T} \int_{\mathbf{R}} u_{x}^{2(2 \ell-2)} u_{x x}^{2} d x d t \\
& \leq C_{0}+C \sup _{t \in[0, T]}\|u(\cdot, t)\|_{L^{\infty}(\mathbf{R})}^{m-1}\left(\|u(\cdot, T)\|_{L^{2}(\mathbf{R})}+\varepsilon \int_{0}^{T} \int_{\mathbf{R}} u_{x}^{2 \ell} d x d t\right)
\end{aligned}
$$


with some $C>0$. In view of an estimate (3.1), we get

$$
\begin{aligned}
& \delta \int_{\mathbf{R}} u_{x}^{2 \ell}(x, T) d x+2 \ell(2 \ell-1) \varepsilon \delta \int_{0}^{T} \int_{\mathbf{R}} u_{x}^{2(2 \ell-2)} u_{x x}^{2} d x d t \\
& \leq C\left(1+\sup _{t \in[0, T]}\|u(\cdot, t)\|_{L^{\infty}(\mathbf{R})}^{m-1}\right)
\end{aligned}
$$

which implies for every $T \in\left(0, T^{*}\right)$ that

$$
\delta^{\frac{1}{2 \ell}}\left\|u_{x}(\cdot, T)\right\|_{L^{2 \ell}(\mathbf{R})} \leq C\left(1+\sup _{t \in[0, T]}\|u(\cdot, t)\|_{L^{\infty}(\mathbf{R})}^{m-1}\right)^{\frac{1}{2 \ell}} \text { with some } C>0 .
$$

Hence by the Hölder's inequality and the estimate (3.1) again, we have for $\forall t_{1} \in[0, T]$

$$
\begin{aligned}
\left|u\left(x, t_{1}\right)\right|^{3} & \leq 3 \int_{-\infty}^{x}\left|u^{2}\left(y, t_{1}\right) u_{x}\left(y, t_{1}\right)\right| d y \\
& \leq 3\left(\int_{\mathbf{R}}|u|^{2 p} d y\right)^{\frac{1}{p}}\left(\int_{\mathbf{R}}\left|u_{x}\right|^{2 \ell} d y\right)^{\frac{1}{2 \ell}} \\
& \leq 3 \delta^{-\frac{1}{2 \ell}}\left(\sup _{t_{1} \in[0, T]}\left\|u\left(\cdot, t_{1}\right)\right\|_{L^{\infty}(\mathbf{R})}^{2 p-2} \int_{\mathbf{R}}|u|^{2} d y\right)^{\frac{1}{p}} \delta \delta^{\frac{1}{2 \ell}}\left\|u_{x}\left(\cdot, t_{1}\right)\right\|_{L^{2 \ell}(\mathbf{R})} \\
& \leq C \delta^{-\frac{1}{2 \ell}} \sup _{t_{1} \in[0, T]}\left\|u\left(\cdot, t_{1}\right)\right\|_{L^{\infty}(\mathbf{R})}^{\frac{2 p-2}{p}}\left(1+\sup _{t_{1} \in[0, T]}\left\|u\left(\cdot, t_{1}\right)\right\|_{L^{\infty}(\mathbf{R})}^{m-1}\right)^{\frac{1}{2 \ell}} \\
& \leq C \delta^{-\frac{1}{2 \ell}} \sup _{t_{1} \in[0, T]}\left\|u\left(\cdot, t_{1}\right)\right\|_{L^{\infty}(\mathbf{R})}^{\frac{1}{\ell}}\left(1+\sup _{t_{1} \in[0, T]}\left\|u\left(\cdot, t_{1}\right)\right\|_{L^{\infty}(\mathbf{R})}^{m-1}\right)^{\frac{1}{2 \ell}}
\end{aligned}
$$

with some $C>0$ where $p=\frac{2 \ell}{2 \ell-1}$. Therefore, for $\forall t \in\left(0, T^{*}\right)$, we have

$$
\sup _{t \in\left(0, T^{*}\right)}\|u(\cdot, t)\|_{L^{\infty}(\mathbf{R})}^{6 \ell} \leq C \delta^{-1} \sup _{t \in\left(0, T^{*}\right)}\|u(\cdot, t)\|_{L^{\infty}(\mathbf{R})}^{2}\left(1+\sup _{t \in\left(0, T^{*}\right)}\|u(\cdot, t)\|_{L^{\infty}(\mathbf{R})}^{m-1}\right)
$$

with some $C>0$. Here we describe $h:=\sup _{t \in\left(0, T^{*}\right)}\|u(\cdot, t)\|_{L^{\infty}(\mathbf{R})}$, and consider the algebraic inequality $h^{6 \ell} \leq C \delta^{-1} h^{2}\left(1+h^{m-1}\right)$. Therefore we obtain the uniform estimate (3.3).

Substituting the uniform boundedness (3.3) of $u$ in the $L^{\infty}$ norm into the inequality (3.4), we can easily obtain 
Lemma 3.3. For any $T \in\left(0, T^{*}\right)$ and $m \in(1,6 \ell-1)(\ell \geq 1)$, there exists a constant $C>0$ such that

$$
\int_{\mathbf{R}} u_{x}(x, T)^{2 \ell} d x+2 \ell(2 \ell-1) \varepsilon \int_{0}^{T} \int_{\mathbf{R}} u_{x}^{2(2 \ell-2)} u_{x x}^{2} d x d t \leq C \delta^{-\frac{2(3 \ell-1)}{6 \ell-m-1}} .
$$

We remark that this inequality implies

$$
\int_{\mathbf{R}} u_{x}(x, T)^{2 \ell} d x+\varepsilon \int_{0}^{T} \int_{\mathbf{R}} u_{x}^{2(2 \ell-2)} u_{x x}^{2} d x d t \leq C \delta^{-\frac{2(3 \ell-1)}{6 \ell-m-1}}
$$

with some $C>0$.

Utilizing some estimates obtained in this section, the uniform boundedness of the sequence $\left\{u^{\varepsilon}\right\}$ in $L^{q}(\mathbf{R})$ for $q \in\left(\frac{3 \ell-1}{\ell}, \frac{3 \ell^{2}+2 \ell-1}{\ell}\right)(\ell \geq 1)$ is established. For some technical reasons, we divide our proof into $\ell>1$ and $\ell=1$.

Proposition 3.1. $\quad$ Suppose the condition (I) for $m<\frac{3 \ell^{2}-q \ell+3 \ell-1}{\ell}(q \in$ $\left.\left(\frac{3 \ell-1}{\ell}, \frac{3 \ell^{2}+2 \ell-1}{\ell}\right), \ell>1\right)$ and the uniform bound (1.6) for the initial data, then the sequence $\left\{u^{\varepsilon}\right\}$ of solutions to Eqs. (1.1), (1.2) is uniformly bounded in $L^{q}(\mathbf{R})$ with respect to $t \in\left(0, T^{*}\right)$ provided that $\delta=O\left(\varepsilon^{\frac{(\ell+1)(6 \ell-m-1)}{2\left(3 \ell^{2}-m \ell-q \ell+3 \ell-1\right)}}\right)$.

Proof. To show the uniform boundedness of the sequence $\left\{u^{\varepsilon}\right\}$ in $L^{q}(\mathbf{R})$, we obtain a priori estimate of the solutions in $L^{q}(\mathbf{R})$. We set $\rho(u):=|u|^{q}$ for $q \in\left(\frac{3 \ell-1}{\ell}, \frac{3 \ell^{2}+2 \ell-1}{\ell}\right)(\ell>1)$. Multiplying Eq. (1.1) by $\rho^{\prime}(u)$ and integrating in space and time, we find

$$
\begin{aligned}
& \int_{\mathbf{R}} \rho(u(x, T)) d x+\varepsilon \int_{0}^{T} \int_{\mathbf{R}} \rho^{\prime \prime}(u) u_{x}^{2 \ell} d x d t \\
& =\int_{\mathbf{R}} \rho\left(u_{0}(x)\right) d x+\delta \int_{0}^{T} \int_{\mathbf{R}} \rho^{\prime}(u)_{x}\left(u_{x}^{2 \ell-1}\right)_{x} d x d t .
\end{aligned}
$$

Applying inequalities (3.1), (3.3) and (3.5), we estimate the second term in the right-hand side of Eq. (3.6):

$$
\begin{aligned}
& \left|\delta \int_{0}^{T} \int_{\mathbf{R}} \rho^{\prime}(u)_{x}\left(u_{x}^{2 \ell-1}\right)_{x} d x d t\right| \\
& \leq\left|(2 \ell-1) \delta \int_{0}^{T} \int_{\mathbf{R}} \rho^{\prime \prime}(u) u_{x} \cdot u_{x}^{2 \ell-2} u_{x x} d x d t\right| \\
& \leq C \delta \int_{0}^{T} \int_{\mathbf{R}}|u|^{q-2}\left|u_{x}\right|\left|u_{x}^{2 \ell-2} u_{x x}\right| d x d t
\end{aligned}
$$




$$
\begin{aligned}
& \leq C \delta\left(\int_{0}^{T} \int_{\mathbf{R}}|u|^{p_{1}(q-2)} d x d t\right)^{\frac{1}{p_{1}}}\left(\int_{0}^{T} \int_{\mathbf{R}}\left|u_{x}\right|^{2 \ell} d x d t\right)^{\frac{1}{2 \ell}}\left(\int_{0}^{T} \int_{\mathbf{R}}\left|u_{x}^{2 \ell-2} u_{x x}\right|^{2} d x d t\right)^{\frac{1}{2}} \\
& \leq C \delta\left(\sup _{t^{\prime} \in(0, T)}\left\|u\left(\cdot, t^{\prime}\right)\right\|_{L^{\infty}(\mathbf{R})}^{p_{1}(q-2)-2} \int_{0}^{T} \int_{\mathbf{R}}|u|^{2} d x d t\right)^{\frac{1}{p_{1}}} \cdot \varepsilon^{-\frac{1}{2 \ell}} \cdot \varepsilon^{-\frac{1}{2}} \delta^{-\frac{3 \ell-1}{6 \ell-m-1}} \\
& \leq C \delta \sup _{t^{\prime} \in(0, T)}\left\|u\left(\cdot, t^{\prime}\right)\right\|_{L^{\infty}(\mathbf{\ell})}^{\frac{\ell(q-3)+1}{\ell}} \cdot T^{\frac{1}{p_{1}}} \cdot \varepsilon^{-\frac{\ell+1}{2 \ell}} \delta^{-\frac{3 \ell-1}{6 \ell-m-1}} \\
& \leq C T^{\frac{\ell-1}{2 \ell}} \varepsilon^{-\frac{\ell+1}{2 \ell}} \delta^{1-\frac{\ell(q-3)+1}{\ell(6 \ell-m-1)}-\frac{3 \ell-1}{6 \ell-m-1}} \\
& \leq C T^{\frac{\ell-1}{2 \ell}} \varepsilon^{-\frac{\ell+1}{2 \ell}} \delta^{\frac{3 \ell^{2}-m \ell-q \ell+3 \ell-1}{\ell(6 \ell-m-1)}}
\end{aligned}
$$

with some $C>0$ where $\frac{1}{p_{1}}+\frac{1}{2 \ell}+\frac{1}{2}=1$ (i.e. $p_{1}=\frac{2 \ell}{\ell-1}$ ) for $\ell>1$. Substituting this estimate into Eq. (3.6), we obtain the uniform estimate in the $L^{q}(\mathbf{R})$ under the condition (I) for $m<\frac{3 \ell^{2}-q \ell+3 \ell-1}{\ell}$. Namely there exists a constant $C>0$ such that, for any $q \in\left(\frac{3 \ell-1}{\ell}, \frac{3 \ell^{2}+2 \ell-1}{\ell}\right)(\ell>1)$,

$$
\sup _{t \in\left(0, T^{*}\right)}\|u(\cdot, t)\|_{L^{q}(\mathbf{R})}^{q} \leq C\left(1+T^{\frac{\ell-1}{2 \ell}} \varepsilon^{-\frac{\ell+1}{2 \ell}} \delta^{\frac{3 \ell^{2}-m \ell-q \ell+3 \ell-1}{\ell(6 \ell-m-1)}}\right) .
$$

From the structure of this a priori estimate (3.7), it follows Proposition 3.1 directly.

When $\ell=1$, we can replace the estimates (3.1), (3.3), (3.5) by following estimates respectively:

$$
\begin{gathered}
\int_{\mathbf{R}} u^{2}(x, T) d x+2 \varepsilon \int_{0}^{T} \int_{\mathbf{R}} u_{x}^{2}(x, t) d x d t \leq C_{0}, \\
\sup _{t \in\left(0, T^{*}\right)}\|u(\cdot, t)\|_{L^{\infty}(\mathbf{R})} \leq C \delta^{-\frac{1}{5-m}}, \\
\int_{\mathbf{R}} u_{x}(x, T)^{2} d x+\varepsilon \int_{0}^{T} \int_{\mathbf{R}} u_{x x}^{2} d x d t \leq C \delta^{-\frac{4}{5-m}} .
\end{gathered}
$$

Hence for $q^{\prime} \in(2,4)$, the uniform boundedness of the sequence $\left\{u^{\varepsilon}\right\}$ of solutions to

$$
\partial_{t} u+\partial_{x} f(u)=\varepsilon \partial_{x}^{2} u-\delta \partial_{x}^{3} u, \quad(x, t) \in \mathbf{R} \times(0, \infty)
$$

in $L^{q^{\prime}}(\mathbf{R})$ is obtained as follows: 
Proposition 3.2. $\quad$ Suppose the condition (I) for $m<5-q^{\prime}\left(q^{\prime} \in(2,4)\right)$ and the uniform bound (1.6) for the initial data, then the sequence $\left\{u^{\varepsilon}\right\}$ of solutions to Eqs. (1.1)', (1.2) is uniformly bounded in $L^{q^{\prime}}(\mathbf{R})$ with respect to $t \in\left(0, T^{*}\right)$ provided that $\delta=O\left(\varepsilon^{\frac{5-m}{5-m-q^{\prime}}}\right)$.

Proof. In the same way of proof of Proposition 3.1, we set $\widetilde{\rho}(u):=|u|^{q^{\prime}}$ for $q^{\prime} \in(2,4)$, and multiply Eq. (1.1)' by $\widetilde{\rho}^{\prime}(u)$. Integrating it in space and time, we get

$$
\begin{aligned}
& \int_{\mathbf{R}} \widetilde{\rho}(u(x, T)) d x+\varepsilon \int_{0}^{T} \int_{\mathbf{R}} \widetilde{\rho}^{\prime \prime}(u) u_{x}^{2} d x d t \\
& =\int_{\mathbf{R}} \widetilde{\rho}\left(u_{0}(x)\right) d x+\delta \int_{0}^{T} \int_{\mathbf{R}} \widetilde{\rho}^{\prime \prime}(u) u_{x} u_{x x} d x d t .
\end{aligned}
$$

Thus using (3.1)', (3.3)' and (3.5)', we can obtain the estimate for the second term in the right-hand side of Eq. (3.8):

$$
\begin{aligned}
& \left|\delta \int_{0}^{T} \int_{\mathbf{R}} \widetilde{\rho}^{\prime \prime}(u) u_{x} u_{x x} d x d t\right| \\
& \leq C \delta \int_{0}^{T} \int_{\mathbf{R}}|u|^{q^{\prime}-2}\left|u_{x}\right|\left|u_{x x}\right| d x d t \\
& \leq C \delta \sup _{t^{\prime} \in(0, T)} \|\left. u\left(\cdot, t^{\prime}\right)\right|_{L^{\infty}(\mathbf{R})} ^{q^{\prime}-2}\left(\int_{0}^{T} \int_{\mathbf{R}}\left|u_{x}\right|^{2}\right)^{\frac{1}{2}}\left(\int_{0}^{T} \int_{\mathbf{R}}\left|u_{x x}\right|^{2}\right)^{\frac{1}{2}} \\
& \leq C \delta \cdot \delta^{-\frac{q^{\prime}-2}{5-m}} \cdot \varepsilon^{-\frac{1}{2}} \cdot \varepsilon^{-\frac{1}{2}} \delta^{-\frac{2}{5-m}} \\
& \leq C \varepsilon^{-1} \delta^{\frac{5-m-q^{\prime}}{5-m}}
\end{aligned}
$$

with some $C>0$. Therefore substituting this estimate into Eq. (3.8), it follows that there exists a constant $C>0$ such that, for any $q^{\prime} \in(2,4)$,

$$
\sup _{t \in\left(0, T^{*}\right)}\|u(\cdot, t)\|_{L^{q^{\prime}}(\mathbf{R})}^{q^{\prime}} \leq C\left(1+\varepsilon^{-1} \delta^{\frac{5-m-q^{\prime}}{5-m}}\right) .
$$

which gives Proposition 3.2 for $m<5-q^{\prime}$.

Combining Propositions 3.1 and 3.2, for any $\ell \geq 1$, we arrive at the uniform boundedness of the sequence $\left\{u^{\varepsilon}\right\}$ in $L^{q}(\mathbf{R})$.

Corollary 3.1. Suppose the condition (I) for $m<\frac{3 \ell^{2}-q \ell+3 \ell-1}{\ell}(q \in$ $\left.\left(\frac{3 \ell-1}{\ell}, \frac{3 \ell^{2}+2 \ell-1}{\ell}\right), \ell \geq 1\right)$ and the uniform bound (1.6) for the initial data, 
then the sequence $\left\{u^{\varepsilon}\right\}$ of solutions to Eqs. (1.1), (1.2) is uniformly bounded in $L^{q}(\mathbf{R})$ with respect to $t \in\left(0, T^{*}\right)$ provided that $\delta=O\left(\varepsilon^{\frac{(\ell+1)(6 \ell-m-1)}{2\left(3 \ell^{2}-m \ell-q \ell+3 \ell-1\right)}}\right)$.

Due to the fact that $\left[\frac{3 \ell^{2}+3 \ell-1}{2 \ell}, 3 \ell\right] \subset\left(\frac{3 \ell-1}{\ell}, \frac{3 \ell^{2}+2 \ell-1}{\ell}\right)(\ell \geq 1)$ and $q>$ $\frac{3 \ell^{2}-q \ell+3 \ell-1}{\ell}(>m)$ for $q \in\left[\frac{3 \ell^{2}+3 \ell-1}{2 \ell}, 3 \ell\right]$, Corollary 3.1 holds for $m<q$ $\left(q \in\left[\frac{3 \ell^{2}+3 \ell-1}{2 \ell}, 3 \ell\right], \ell \geq 1\right)$.

\section{$\S 4 . \quad$ Proof of the Main Result}

Due to the uniform boundedness of a sequence $\left\{u^{\varepsilon}\right\}$ in $L^{\infty}\left(0, T^{*} ; L^{q}(\mathbf{R})\right)$ (Corollary 3.1) in the previous section, we can apply Theorem 2.1 as a convergence tool if it is obtained that a Young measure $\nu$ associated with a sequence $\left\{u^{\varepsilon}\right\}$ is an entropy m.-v. solution of the Cauchy problem (1.3), (1.4). To accomplish the objective, we show the proof of the main Theorem 1.1 by using several uniform estimates for the sequence $\left\{u^{\varepsilon}\right\}$ under the growth condition (I) for $m<q\left(q \in\left[\frac{3 \ell^{2}+3 \ell-1}{2 \ell}, 3 \ell\right]\right)$ and the assumptions (1.5), (1.6) for the initial data $u_{0}^{\varepsilon}$.

Proof of Theorem 1.1. To apply the convergence Theorem 2.1, we will show that a Young measure $\nu$ associated with a sequence $\left\{u^{\varepsilon}\right\}$ is an entropy m.-v. solution. In other words, it is necessary to establish that a Young measure $\nu$ satisfies the entropy inequality (2.2) and the initial condition (2.3).

As first step, we consider for the entropy inequality (2.2). For any convex smooth function $\eta(u): \mathbf{R} \rightarrow \mathbf{R}$ such that $\eta^{\prime}$ and $\eta^{\prime \prime}$ are uniformly bounded on $\mathbf{R}$, we consider the distribution

$$
\Lambda^{\varepsilon}:=\partial_{t} \eta\left(u^{\varepsilon}\right)+\partial_{x} \sigma\left(u^{\varepsilon}\right),
$$

where the flux $\sigma: \mathbf{R} \rightarrow \mathbf{R}$ is defined by $\sigma^{\prime}(u)=f^{\prime}(u) \eta^{\prime}(u)$ for $u \in \mathbf{R}$. Then $\Lambda^{\varepsilon}$ converges to a nonpositive measure in $\mathcal{D}^{\prime}\left(\mathbf{R} \times\left(0, T^{*}\right)\right)$. In fact, we observe that $\Lambda^{\varepsilon}$ is decomposed as follows:

$$
\begin{aligned}
\Lambda^{\varepsilon}= & \eta\left(u^{\varepsilon}\right)_{t}+\sigma\left(u^{\varepsilon}\right)_{x} \\
= & \eta^{\prime}\left(u^{\varepsilon}\right) u_{t}^{\varepsilon}+\sigma^{\prime}\left(u^{\varepsilon}\right) u_{x}^{\varepsilon} \\
= & \eta^{\prime}\left(u^{\varepsilon}\right)\left\{\varepsilon\left(\left(u_{x}^{\varepsilon}\right)^{2 \ell-1}\right)_{x}-\delta\left(\left(u_{x}^{\varepsilon}\right)^{2 \ell-1}\right)_{x x}-f\left(u^{\varepsilon}\right)_{x}\right\}+f^{\prime}\left(u^{\varepsilon}\right) \eta^{\prime}\left(u^{\varepsilon}\right) u_{x}^{\varepsilon} \\
= & \varepsilon \eta^{\prime}\left(u^{\varepsilon}\right)\left(\left(u_{x}^{\varepsilon}\right)^{2 \ell-1}\right)_{x}-\delta \eta^{\prime}\left(u^{\varepsilon}\right)\left(\left(u_{x}^{\varepsilon}\right)^{2 \ell-1}\right)_{x x} \\
= & \varepsilon\left\{\left(\eta^{\prime}\left(u^{\varepsilon}\right)\left(u_{x}^{\varepsilon}\right)^{2 \ell-1}\right)_{x}-\eta^{\prime \prime}\left(u^{\varepsilon}\right)\left(u_{x}^{\varepsilon}\right)^{2 \ell}\right\} \\
& -\delta\left\{\left(\eta^{\prime}\left(u^{\varepsilon}\right)\left(\left(u_{x}^{\varepsilon}\right)^{2 \ell-1}\right)_{x}\right)_{x}-\eta^{\prime \prime}\left(u^{\varepsilon}\right)\left(\left(u_{x}^{\varepsilon}\right)^{2 \ell-1}\right)_{x} u_{x}^{\varepsilon}\right\}
\end{aligned}
$$




$$
\begin{aligned}
= & \varepsilon\left(\eta^{\prime}\left(u^{\varepsilon}\right)\left(u_{x}^{\varepsilon}\right)^{2 \ell-1}\right)_{x}-\varepsilon \eta^{\prime \prime}\left(u^{\varepsilon}\right)\left(u_{x}^{\varepsilon}\right)^{2 \ell} \\
& -\delta\left(\eta^{\prime}\left(u^{\varepsilon}\right)\left(u_{x}^{\varepsilon}\right)^{2 \ell-1}\right)_{x x}+\delta\left(\eta^{\prime \prime}\left(u^{\varepsilon}\right)\left(u_{x}^{\varepsilon}\right)^{2 \ell}\right)_{x}+\delta \eta^{\prime \prime}\left(u^{\varepsilon}\right)\left(\left(u_{x}^{\varepsilon}\right)^{2 \ell-1}\right)_{x} u_{x}^{\varepsilon} \\
= & : \Lambda_{1}+\Lambda_{2}+\Lambda_{3}+\Lambda_{4}+\Lambda_{5} .
\end{aligned}
$$

The estimate of each terms in $\Lambda^{\varepsilon}$ hold for all smooth function $\theta \in C_{0}^{\infty}(\mathbf{R} \times$ $\left.\left(0, T^{*}\right)\right)(\theta \geq 0)$ below. Throughout a process of calculation, we omit the upper-index $\varepsilon$ for simplicity similarly to Section 3 .

To begin with, consider the term $\Lambda_{1}$. By an estimate (3.1) and Hölder's inequality, we have

$$
\begin{aligned}
\left|\left\langle\Lambda_{1}, \theta\right\rangle\right| & =\left|\varepsilon \int_{0}^{T^{*}} \int_{\mathbf{R}} \eta^{\prime}(u) u_{x}^{2 \ell-1} \theta_{x} d x d t\right| \\
& \leq C \varepsilon\left(\iint_{\operatorname{supp} \theta}\left|u_{x}\right|^{p_{1}(2 \ell-1)} d x d t\right)^{\frac{1}{p_{1}}}\left\|\theta_{x}\right\|_{L^{p_{1}^{\prime}}\left(\mathbf{R} \times\left(0, T^{*}\right)\right)} \\
& \leq C \varepsilon\left(\iint_{\operatorname{supp} \theta}\left|u_{x}\right|^{2 \ell} d x d t\right)^{\frac{2 \ell-1}{2 \ell}}\left\|\theta_{x}\right\|_{L^{p_{1}^{\prime}}\left(\mathbf{R} \times\left(0, T^{*}\right)\right)} \\
& \leq C \varepsilon \cdot \varepsilon^{-\frac{2 \ell-1}{2 \ell}} \\
& \leq C \varepsilon^{\frac{1}{2 \ell}} \rightarrow 0(\varepsilon \rightarrow 0)
\end{aligned}
$$

with some $C>0$ where $\frac{1}{p_{1}}+\frac{1}{p_{1}^{\prime}}=1$ with $p_{1}(2 \ell-1)=2 \ell$. We denotes by $\operatorname{supp} \theta$ the support of $\theta$ in $\mathbf{R} \times\left(0, T^{*}\right)$.

Next the second term $\Lambda_{2}$ is nonpositive:

$$
\left\langle\Lambda_{2}, \theta\right\rangle=-\varepsilon \int_{0}^{T^{*}} \int_{\mathbf{R}} \eta^{\prime \prime}(u) u_{x}^{2 \ell} \theta d x d t \leq 0 .
$$

Using an estimate (3.1) again, we estimate the term $\Lambda_{3}$ :

$$
\begin{aligned}
\left|\left\langle\Lambda_{3}, \theta\right\rangle\right| & =\left|\delta \int_{0}^{T^{*}} \int_{\mathbf{R}} \eta^{\prime}(u) u_{x}^{2 \ell-1} \theta_{x x} d x d t\right| \\
& \leq C \delta\left(\iint_{\operatorname{supp} \theta}\left|u_{x}\right|^{p_{2}(2 \ell-1)} d x d t\right)^{\frac{1}{p_{2}}}\left\|\theta_{x x}\right\|_{L^{p_{2}^{\prime}\left(\mathbf{R} \times\left(0, T^{*}\right)\right)}} \\
& \leq C \delta\left(\iint_{\operatorname{supp} \theta}\left|u_{x}\right|^{2 \ell} d x d t\right)^{\frac{2 \ell-1}{2 \ell}}\left\|\theta_{x x}\right\|_{L^{p_{2}^{\prime}\left(\mathbf{R} \times\left(0, T^{*}\right)\right)}} \\
& \leq C \varepsilon^{-\frac{2 \ell-1}{2 \ell} \delta}
\end{aligned}
$$

with some $C>0$ where $\frac{1}{p_{2}}+\frac{1}{p_{2}^{\prime}}=1$ with $p_{2}(2 \ell-1)=2 \ell$. In this case, when $\delta=o\left(\varepsilon^{\frac{2 \ell-1}{2 \ell}}\right), \Lambda_{3} \rightarrow 0$ in $\mathcal{D}^{\prime}\left(\mathbf{R} \times\left(0, T^{*}\right)\right)$ as $\varepsilon \rightarrow 0$. 
On the other hand, by applying an estimate (3.1) to $\Lambda_{4}$, we find:

$$
\begin{aligned}
\left|\left\langle\Lambda_{4}, \theta\right\rangle\right| & =\left|\delta \int_{0}^{T^{*}} \int_{\mathbf{R}} \eta^{\prime \prime}(u) u_{x}^{2 \ell} \theta_{x} d x d t\right| \\
& \leq C \delta\left\|\theta_{x x}\right\|_{L^{\infty}\left(\mathbf{R} \times\left(0, T^{*}\right)\right)} \int_{0}^{T^{*}} \int_{\mathbf{R}}\left|u_{x}\right|^{2 \ell} d x d t \\
& \leq C \varepsilon^{-1} \delta
\end{aligned}
$$

with some $C>0$ which includes $\delta=o(\varepsilon)$.

To deal with the last term $\Lambda_{5}$, we divide into $\ell>1$ and $\ell=1$. In the case that $\ell>1$, remarking that $\left(u_{x}^{2 \ell-1}\right)_{x}=u_{x}^{2 \ell-2} u_{x x}$, we combine the estimates (3.1) and (3.5) as follows:

$$
\begin{aligned}
\left|\left\langle\Lambda_{5}, \theta\right\rangle\right|= & \left|\delta \int_{0}^{T^{*}} \int_{\mathbf{R}} \eta^{\prime \prime}(u)\left(u_{x}^{2 \ell-1}\right)_{x} u_{x} \theta d x d t\right| \\
\leq & C \delta\|\theta\|_{L^{p_{3}}\left(\mathbf{R} \times\left(0, T^{*}\right)\right)} \\
& \times\left(\iint_{\operatorname{supp} \theta}\left|u_{x}^{2 \ell-2} u_{x x}\right|^{2} d x d t\right)^{\frac{1}{2}}\left(\iint_{\operatorname{supp} \theta}\left|u_{x}\right|^{2 \ell} d x d t\right)^{\frac{1}{2 \ell}} \\
\leq & C \delta \cdot \varepsilon^{-\frac{1}{2}} \delta^{-\frac{3 \ell-1}{6 \ell-m-1}} \cdot \varepsilon^{-\frac{1}{2 \ell}} \\
\leq & C \varepsilon^{-\frac{\ell+1}{2 \ell}} \delta^{\frac{3 \ell-m}{6 \ell-m-1}}
\end{aligned}
$$

with some $C>0$ where $\frac{1}{2}+\frac{1}{2 \ell}+\frac{1}{p_{3}}=1$ hence $p_{3}=\frac{2 \ell}{\ell-1}(\ell>1)$. In the case that $\ell=1$, using the estimates (3.1)' and (3.5)', it follows that

$$
\begin{aligned}
\left|\left\langle\Lambda_{5}, \theta\right\rangle\right|= & \left|\delta \int_{0}^{T^{*}} \int_{\mathbf{R}} \eta^{\prime \prime}(u) u_{x} u_{x x} \theta d x d t\right| \\
\leq & \left.C \delta|| \theta \|_{L^{\infty}\left(\mathbf{R} \times\left(0, T^{*}\right)\right)}\left|u_{x}\right|^{2} d x d t\right)^{\frac{1}{2}}\left(\iint_{\operatorname{supp} \theta}\left|u_{x x}\right|^{2} d x d t\right)^{\frac{1}{2}} \\
& \times\left(\iint_{\operatorname{supp} \theta}\right. \\
\leq & C \delta \cdot \varepsilon^{-\frac{1}{2}} \cdot \varepsilon^{-\frac{1}{2}} \delta^{-\frac{2}{5-m}} \\
\leq & C \varepsilon^{-1} \delta^{\frac{3-m}{5-m}}
\end{aligned}
$$

with some $C>0$. Now paying attention to an exponent of $\delta$ which are yielded from inequalities (4.6) and (4.7), it holds that $\frac{3 \ell-m}{6 \ell-m-1}>0$ for $m<q$ $\left(q \in\left[\frac{3 \ell^{2}+3 \ell-1}{2 \ell}, 3 \ell\right], \ell \geq 1\right)$. Hence inequalities (4.6) and (4.7) imply the condition $\delta=o\left(\varepsilon^{\frac{(\ell+1)(6 \ell-m-1)}{2 \ell(3 \ell-m)}}\right)$. 
By the estimates $(4.2)-(4.7)$, if $\delta=o\left(\varepsilon^{\frac{(\ell+1)(6 \ell-m-1)}{2 \ell(3 \ell-m)}}\right)$, then $\Lambda^{\varepsilon}$ converges to a nonpositive measure in $\mathcal{D}^{\prime}\left(\mathbf{R} \times\left(0, T^{*}\right)\right)$ as $\varepsilon \rightarrow 0$. In particular, one can verify that $\delta=O\left(\varepsilon^{\frac{(\ell+1)(6 \ell-m-1)}{2\left(3 \ell^{2}-m \ell-q \ell+3 \ell-1\right)}}\right)$ implies $\delta=o\left(\varepsilon^{\frac{(\ell+1)(6 \ell-m-1)}{2 \ell(3 \ell-m)}}\right)$. Combining the convergence property of $\Lambda^{\varepsilon}$ and that $\eta(u) \rightarrow\langle\nu, \eta\rangle, \sigma(u) \rightarrow\langle\nu, \sigma\rangle$ in $\mathcal{D}^{\prime}(\mathbf{R} \times$ $\left.\left(0, T^{*}\right)\right)$ as $\varepsilon \rightarrow 0$ which are obtained by owing to Proposition 2.1, it follows that

$$
\partial_{t}\left\langle\nu_{(x, t)}(\lambda), \eta(\lambda)\right\rangle+\partial_{x}\left\langle\nu_{(x, t)}(\lambda), \sigma(\lambda)\right\rangle \leq 0
$$

for any convex entropy pairs such that $\eta^{\prime}$ and $\eta^{\prime \prime}$ are uniformly bounded on $\mathbf{R}$. Therefore, by the regularization of $|u-k|$ (for all $k \in \mathbf{R}$ ), the inequality (2.2) follows.

Next, in the rest of this paper, we give a proof that the initial condition (2.3) is satisfied by the argument due to DiPerna [8] and Szepessy [27].

Let $g$ be a function $g(\lambda)=|\lambda|^{r}$ for $r \in(1,2)$ and $\left\{\phi_{n}\right\} \subseteq C_{0}^{\infty}(\mathbf{R})$ be a sequence of test functions such that

$$
\lim _{n \rightarrow \infty} \phi_{n}=g^{\prime}\left(u_{0}\right) \quad \text { in } \quad L^{r^{\prime}}(\mathbf{R})
$$

where $\frac{1}{r}+\frac{1}{r^{\prime}}=1$. Furthermore we set

$$
G\left(\lambda, \lambda_{0}\right):=g(\lambda)-g\left(\lambda_{0}\right)-g^{\prime}\left(\lambda_{0}\right)\left(\lambda-\lambda_{0}\right)
$$

Following a detailed discussion in $[9,22]$, by the Cauchy-Schwarz inequality and the Jensen inequality, we can easily check

$$
\begin{aligned}
& \left(\frac{1}{T} \int_{0}^{T} \int_{K}\left\langle\nu_{(x, t)}(\lambda),\left|\lambda-u_{0}(x)\right|\right\rangle d x d t\right)^{2} \\
& \leq \frac{C_{K}}{T} \int_{0}^{T} \int_{K}\left\langle\nu_{(x, t)}(\lambda), G\left(\lambda, u_{0}(x)\right)\right\rangle d x d t \\
& \leq \frac{C_{K}}{T} \int_{0}^{T} \int_{\mathbf{R}}\left\langle\nu_{(x, t)}(\lambda), u_{0}(x)-\lambda\right\rangle \phi_{n} d x d t \\
& \quad+C_{K}\left\|u_{0}\right\|_{L^{r}(\mathbf{R})}\left\|g^{\prime}\left(u_{0}\right)-\phi_{n}\right\|_{L^{r^{\prime}}(\mathbf{R})}
\end{aligned}
$$

for any compact set $K \subseteq \mathbf{R}$. From the definition of $\phi_{n}$, it follows that

$$
\left\|g^{\prime}\left(u_{0}\right)-\phi_{n}\right\|_{L^{r^{\prime}}(\mathbf{R})} \rightarrow 0 \text { as } n \rightarrow \infty
$$

which indicates that the second term in the right-hand side of the inequality (4.9) tends to zero as $n \rightarrow \infty$. Consequently, it is sufficient to show that the 
first term of the right-hand side of Eq. (4.9) tends to zero as the upper bound at $t=0$ i.e.

$$
\lim _{T \rightarrow 0^{+}} \frac{1}{T} \int_{0}^{T} \int_{\mathbf{R}}\left\langle\nu_{(x, t)}(\lambda), u_{0}(x)-\lambda\right\rangle \phi_{n} d x d t \leq 0
$$

so as to prove the initial condition (2.3). From the definition of the Young measure $\nu$, it holds that

$$
\begin{aligned}
\frac{1}{T} \int_{0}^{T} \int_{\mathbf{R}}\left\langle\nu_{(x, t)}(\lambda), u_{0}(x)-\lambda\right\rangle \phi_{n} d x d t \\
=\lim _{\varepsilon \rightarrow 0} \frac{1}{T} \int_{0}^{T} \int_{\mathbf{R}}\left(u_{0}(x)-u^{\varepsilon}(x, t)\right) \phi_{n} d x d t \\
=\lim _{\varepsilon \rightarrow 0} \frac{1}{T} \int_{0}^{T} \int_{\mathbf{R}} u_{0}(x) \phi_{n} d x d t-\lim _{\varepsilon \rightarrow 0} \frac{1}{T} \int_{0}^{T} \int_{\mathbf{R}} u^{\varepsilon}(x, t) \phi_{n} d x d t \\
=\lim _{\varepsilon \rightarrow 0}\left(\int_{\mathbf{R}}\left(u_{0}(x)-u_{0}^{\varepsilon}(x)\right) \phi_{n} d x+\int_{\mathbf{R}} u_{0}^{\varepsilon}(x) \phi_{n} d x\right) \\
-\lim _{\varepsilon \rightarrow 0} \frac{1}{T} \int_{0}^{T} \int_{\mathbf{R}} u^{\varepsilon}(x, t) \phi_{n} d x d t \\
=\lim _{\varepsilon \rightarrow 0} \int_{\mathbf{R}}\left(u_{0}(x)-u_{0}^{\varepsilon}(x)\right) \phi_{n} d x-\lim _{\varepsilon \rightarrow 0} \frac{1}{T} \int_{0}^{T} \int_{\mathbf{R}}\left(u^{\varepsilon}(x, t)-u_{0}^{\varepsilon}(x)\right) \phi_{n} d x d t \\
=-\lim _{\varepsilon \rightarrow 0} \frac{1}{T} \int_{0}^{T} \int_{\mathbf{R}}\left(\int_{0}^{t} \partial_{s} u^{\varepsilon}(x, s) d s\right) \phi_{n}(x) d x d t .
\end{aligned}
$$

where we use an assumption (1.5) for the initial data. Here, by the growth condition (I) and the definition of $\phi_{n}$, we remark that $|f(u)| \leq C\left(|u|+|u|^{m}\right)$ $(C>0)$ and $\int_{\mathbf{R}} \phi_{n} d x<C_{n}$, and set

$$
\Gamma^{\varepsilon}:=\frac{1}{T} \int_{0}^{T} \int_{\mathbf{R}}\left(\int_{0}^{t} \partial_{s} u^{\varepsilon}(x, s) d s\right) \phi_{n}(x) d x d t .
$$

Owing to the uniform boundedness of a sequence $\left\{u^{\varepsilon}\right\}$ in $L^{\infty}\left(0, T^{*} ; L^{2}(\mathbf{R}) \cap\right.$ $L^{q}(\mathbf{R})$ ) for $q>m$ (an estimate (3.1), Corollary 3.1) and the same argument as the inequalities $(4.2),(4.4)$, we can estimate $\Gamma^{\varepsilon}$ as follows:

$$
\begin{aligned}
& \left|\Gamma^{\varepsilon}\right| \\
& =\left|\frac{1}{T} \int_{0}^{T} \int_{\mathbf{R}}\left(\int_{0}^{t} \partial_{s} u^{\varepsilon}(x, s) d s\right) \phi_{n}(x) d x d t\right| \\
& =\left|\frac{1}{T} \int_{0}^{T} \int_{\mathbf{R}}\left(\int_{0}^{t}\left(-\partial_{x} f\left(u^{\varepsilon}\right)+\varepsilon \partial_{x}\left(u_{x}^{\varepsilon}\right)^{2 \ell-1}-\delta \partial_{x}^{2}\left(u_{x}^{\varepsilon}\right)^{2 \ell-1}\right) d s\right) \phi_{n}(x) d x d t\right|
\end{aligned}
$$




$$
\begin{aligned}
= & \left|\frac{1}{T} \int_{0}^{T} \int_{\mathbf{R}} \int_{0}^{t}\left(f\left(u^{\varepsilon}\right) \partial_{x} \phi_{n}-\varepsilon\left(u_{x}^{\varepsilon}\right)^{2 \ell-1} \partial_{x} \phi_{n}-\delta\left(u_{x}^{\varepsilon}\right)^{2 \ell-1} \partial_{x}^{2} \phi_{n}\right) d s d x d t\right| \\
\leq & \frac{C}{T} \int_{0}^{T} \int_{\mathbf{R}} \int_{0}^{t}\left(\left|u^{\varepsilon}\right|+\left|u^{\varepsilon}\right|^{m} \mid\right)\left|\partial_{x} \phi_{n}\right| d s d x d t+C \varepsilon^{\frac{1}{2 \ell}}+C \varepsilon^{-\frac{2 \ell-1}{2 \ell}} \delta \\
\leq & \frac{C}{T} \int_{0}^{T} d t \int_{0}^{t} d s\left(\int_{\mathbf{R}}\left|u^{\varepsilon}\right|^{2} d x\right)^{\frac{1}{2}}\left(\int_{\mathbf{R}}\left|\partial_{x} \phi_{n}\right|^{2} d x\right)^{\frac{1}{2}} \\
& +\frac{C}{T} \int_{0}^{T} d t \int_{0}^{t} d s\left(\int_{\mathbf{R}}\left|u^{\varepsilon}\right|^{m \tilde{q}} d x\right)^{\frac{1}{\tilde{q}}}\left(\int_{\mathbf{R}}\left|\partial_{x} \phi_{n}\right|^{\tilde{q}^{\prime}} d x\right)^{\frac{1}{\tilde{q}^{\prime}}} \\
& +C \varepsilon^{\frac{1}{2 \ell}}+C \varepsilon^{-\frac{2 \ell-1}{2 \ell} \delta} \\
\leq & C_{n_{1}} T+\frac{C}{T} \cdot C_{n_{2}} \int_{0}^{T} d t \int_{0}^{t} d s\left(\int_{\mathbf{R}}\left|u^{\varepsilon}\right|^{q} d x\right)^{\frac{1}{\tilde{q}}}+C \varepsilon^{\frac{1}{2 \ell}}+C \varepsilon^{-\frac{2 \ell-1}{2 \ell}} \delta \\
\leq & C_{n} T+C \varepsilon^{\frac{1}{2 \ell}}+C \varepsilon^{-\frac{2 \ell-1}{2 \ell} \delta}
\end{aligned}
$$

with some $C>0$ where $\frac{1}{\tilde{q}}+\frac{1}{\tilde{q}^{\prime}}=1$ with $m \tilde{q}=q(>m)$. When $\varepsilon \rightarrow 0$ with $\delta=o\left(\varepsilon^{\frac{2 \ell-1}{2 \ell}}\right)$, we obtain that $\limsup _{\varepsilon \rightarrow 0}\left|\Gamma^{\varepsilon}\right| \leq C_{n} T$. Hence we arrive at

$$
\frac{1}{T} \int_{0}^{T} \int_{\mathbf{R}}\left\langle\nu_{(x, t)}(\lambda), u_{0}(x)-\lambda\right\rangle \phi_{n} d x d t \leq C_{n} T,
$$

which implies the inequality (4.10), accordingly, we establish that the initial condition (2.3) is satisfied.

Consequently Young measure $\nu$ is an entropy m.-v. solution to Eqs. (1.3) and (1.4). Applying Theorem 2.1, the sequence $\left\{u^{\varepsilon}\right\}$ of solutions to Eqs. (1.1) and (1.2) converges to the unique entropy solution $u \in L^{\infty}\left(0, T^{*} ; L^{q}(\mathbf{R})\right)$ to Eqs. (1.3) and (1.4) in $L^{k}\left(0, T^{*} ; L^{p}(\mathbf{R})\right)(\forall k<\infty$ and $\forall p<q)$. This completes the proof of Theorem 1.1.

\section{References}

[1] P. Baiti, P. G. LeFloch and B. Piccoli, Uniqueness of classical and nonclassical solutions for nonlinear hyperbolic systems, J. Differential Equations 172 (2001), no. 1, 59-82.

[2] N. Bedjaoui and P. G. LeFloch, Diffusive-dispersive traveling waves and kinetic relations. I. Nonconvex hyperbolic conservation laws, J. Differential Equations 178 (2002), no. 2, $574-607$

[3] J. L. Bona and M. E. Schonbek, Travelling-wave solutions to the Korteweg-de VriesBurgers equation, Proc. Roy. Soc. Edinburgh Sect. A 101 (1985), no. 3-4, 207-226.

[4] J. L. Bona and R. Smith, The initial-value problem for the Korteweg-de Vries equation, Philos. Trans. Roy. Soc. London Ser. A 278 (1975), no. 1287, 555-601.

[5] G. Q. Chen and Y. G. Lu, A study of approaches to applying the theory of compensated compactness, Kexue Tongbao (Chinese) 33 (1988), no. 9, 641-644. 
[6] G. Q. Chen and Y. G. Lu, Convergence of the approximate solutions to isentropic gas dynamics, Acta Math. Sci., (English Ed.) 10 (1990), no. 1, 39-45.

[7] G. M. Coclite and K. H. Karlsen, A Singular limit problem for conservation laws related to the Camassa-Holm shallow water equation, Comm. Partial Differential Equations 31 (2006), no. 8, 1253-1272.

[8] R. J. DiPerna, Measure-valued solutions to conservation laws, Arch. Rational Mech. Anal. 88 (1985), no. 3, 223-270.

[9] N. Fujino and M. Yamazaki, Hyperbolic conservation laws with nonlinear diffusion and nonlinear dispersion, J. Differential Equations 228 (2006), no. 1, 171-190.

[10] B. T. Hayes and P. G. LeFloch, Non-classical shocks and kinetic relations: scalar conservation laws, Arch. Rational Mech. Anal. 139 (1997), no. 1, 1-56.

[11] Nonclassical shocks and kinetic relations: strictly hyperbolic systems, SIAM J. Math. Anal. 31 (2000), no. 5, 941-991 (electronic).

[12] Nonclassical shocks and kinetic relations: finite difference schemes, SIAM J. Numer. Anal. 35 (1998), no. 6, 2169-2194 (electronic).

[13] E. Hopf, The partial differential equation $u_{t}+u u_{x}=\mu u_{x x}$, Comm. Pure Appl. Math. 3 (1950), 201-230.

[14] D. Jacobs, B. McKinney and M. Shearer, Travelling wave solutions of the modified Korteweg-de Vries-Burgers equation, J. Differential Equations 116 (1995), no. 2, 448 467.

[15] C. I. Kondo and P. G. Lefloch, Zero diffusion-dispersion limits for scalar conservation laws, SIAM J. Math. Anal. 33 (2002), no. 6, 1320-1329 (electronic).

[16] D. J. Korteweg and G. de Vries, On the change of form of long waves advancing in a rectangular canal, and on a new type of long stationary waves, Philosophical Magazine 39 (1895), 422-443.

[17] S. N. Kružkov, First order quasilinear equations in several independent variables, Mat. Sb. (N.S.) 81 (123) (1970), 228-255; Math. USSR Sb. 10 (1970), 217-243.

[18] P. D. Lax, The zero dispersion limit, a deterministic analogue of turbulence, Comm. Pure Appl. Math. 44 (1991), no. 8-9, 1047-1056.

[19] P. D. Lax and C. D. Levermore, The small dispersion limit of the Korteweg-de Vries equation. II-III, Comm. Pure Appl. Math. 36 (1983), no. 3, 253-290, no, 5, 571-593, no. $6,809-829$.

[20] The zero dispersion limit for the Korteweg-de Vries KdV equation, Proc. Nat. Acad. Sci. U.S.A. 76 (1979), no. 8, 3602-3606.

[21] P. G. LeFloch, An introduction to nonclassical shocks of systems of conservation laws, in An introduction to recent developments in theory and numerics for conservation laws (Freiburg/Littenweiler, 1997), 28-72, Springer, Berlin.

[22] P. G. LeFloch and R. Natalini, Conservation laws with vanishing nonlinear diffusion and dispersion, Nonlinear Anal. 36 (1999), no. 2, Ser. A: Theory Methods, 213-230.

[23] P. G. Lefloch and C. Rohde, High-order schemes, entropy inequalities, and nonclassical shocks, SIAM J. Numer. Anal. 37 (2000), no. 6, 2023-2060 (electronic).

[24] P. G. LeFloch and M. D. Thanh, Nonclassical Riemann solvers and kinetic relations. III. A nonconvex hyperbolic model for van der Waals fluids, Electron. J. Differential Equations 2000, No. 72, 1-19. (electronic).

[25] Y. Lu, Hyperbolic conservation laws and the compensated compactness method, Chapman \& Hall/CRC, Boca Raton, FL, 2003.

[26] M. E. Schonbek, Convergence of solutions to nonlinear dispersive equations, Comm. Partial Differential Equations 7 (1982), no. 8, 959-1000.

[27] A. Szepessy, An existence result for scalar conservation laws using measure valued solutions, Comm. Partial Differential Equations 14 (1989), no. 10, 1329-1350. 